\title{
Local measurements of velocity fluctuations and diffusion coefficients for a granular material flow
}

\author{
By V. V. R. NATARAJAN, M. L. HUNT AND E. D. TAYLOR \\ Division of Engineering and Applied Sciences, California Institute of Technology, \\ Pasadena, CA 91125, USA
}

(Received 24 August 1994 and in revised form 24 April 1995)

Measurements were made of two components of the average and fluctuating velocities, and of the local self-diffusion coefficients in a flow of granular material. The experiments were performed in a $1 \mathrm{~m}$-high vertical channel with roughened sidewalls and with polished glass plates at the front and the back to create a two-dimensional flow. The particles used were glass spheres with a nominal diameter of $3 \mathrm{~mm}$. The flows were high density and were characterized by the presence of long-duration frictional contacts between particles. The velocity measurements indicated that the flows consisted of a central uniform regime and a shear regime close to the walls. The fluctuating velocities in the transverse direction increased in magnitude from the centre towards the walls. A similar variation was not observed for the streamwise fluctuations. The self-diffusion coefficients showed a significant dependence on the fluctuating velocities and the shear rate. The velocity fluctuations were highly anistropic with the streamwise components being 2 to 2.5 times the transverse components. The selfdiffusion coefficients for the streamwise direction were an order-of-magnitude higher than those for the transverse direction. The surface roughness of the particles led to a decrease in the self-diffusion coefficients.

\section{Introduction}

The term 'granular material flow' is applied in the literature to particulate flows such as the flow of coal down an inclined chute, the discharge of grains from a hopper or the motion of debris in a landslide. In these flows, the material has an overall bulk motion; however, individual particles may collide, roll or slide against each other, and may interact with the bounding surfaces. Hence, the individual particle motions are composed of a mean component and a fluctuating, or random, component. An analogy is drawn between this random motion and the random motion of molecules. As a result, much of the theoretical analysis of these flows has developed from concepts derived from the dense-gas kinetic theory (Savage \& Jeffrey 1981; Jenkins \& Savage 1983; Lun et al. 1984) and molecular dynamics simulations (Campbell 1989; Walton \& Braun 1986; Dai 1993).

In the literature, the term 'granular temperature' is used to quantify the random motions of particles about the mean velocity, and is defined as the average of the sum of the squares of the three fluctuating velocity components. Since the granular temperature is a measure of the specific random kinetic energy of the flow, it replaces the thermodynamic temperature in the dense-gas kinetic theory analysis. Although the granular temperature is a key property in the analytical studies, there have been few attempts to measure its magnitude in experimental studies. Ahn, Brennen \& Sabersky (1991) and Hsiau \& Hunt (1993a) only measured the streamwise fluctuation velocity 
component using fibre-optic probes. Drake (1991) measured both fluctuation velocity components in a two-dimensional, high-shear-rate low-density flow down an inclined chute, using high-speed photographic techniques. The latter work also presented evidence that the fluctuation velocities were anisotropic.

Another area of considerable industrial interest is particle mixing in monodisperse and polydisperse flows. Because of the random component of particle motion, the particles can exhibit a diffusive motion similar to that found in gases and liquids. Studies based on kinetic theories by Savage \& Dai (1993) and by Hsiau \& Hunt (1993b) define a granular self-diffusion coefficient that is proportional to the square root of the granular temperature and the particle diameter and is analogous to the corresponding coefficient for gaseous self-diffusion (Chapman \& Cowling 1971). Studies based on kinetic theory show a strong dependence of the granular temperature on shear rates, and therefore the self-diffusion coefficients are expected to be functions of shear rates as well.

Several experimental studies have sought to investigate this relation between shear rates and self-diffusion coefficients. Hwang \& Hogg (1980) examined the dependence of the transverse diffusion coefficients on the shear rate for flows in an inclined chute. Scott \& Bridgewater (1976) studied the diffusive process in a simple shear cell subjected to reciprocating strains. Their results were later corrected by Bridgewater (1980) by appropriately accounting for Taylor dispersion effects. Buggish \& Loffelmann (1989) investigated the effect of shear on diffusion in an assembly of polydisperse vertical cylindrical rods in a Couette apparatus. Zik \& Stavens (1991) conducted experiments on a vertically vibrated layer of grains, and measured diffusive displacements in the transverse direction. A similar vibratory experiment was also performed by Hunt, Hsiau \& Hong (1994). This experiment demonstrated that, for thin beds, the particle mixing in the direction of vibration was diffusive and could be modelled using the diffusion coefficient as defined by Savage \& Dai (1993) and Hsiau \& Hunt (1993b). All these experiments indicated that granular mixing could be modelled as a simple selfdiffusive process. However, none of these experiments made actual measurements of the fluctuation velocities. Savage \& Dai (1993), Campbell (1993) and Dai (1993) conducted computer simulations based on molecular dynamics models to examine selfdiffusion in simple unbounded shear flows. Dai (1993) considered the effect of particle surface roughness.

The present work is motivated by an interest in examining self-diffusion in granular flows, specifically with respect to shear rates and fluctuation velocities. Hsiau \& Hunt (1993a) studied the evolution of the mixing layer at the centre in a vertical channel flow. They examined the influence of different wall conditions, chute widths and particle diameters on the diffusive process and measured the streamwise mean and fluctuation velocity profiles using fibre-optic probes. However, they did not make any measurements of the transverse fluctuation velocities. Furthermore, their diffusion studies were confined to the central uniform flow regime, and hence relationships between the shear rates and the diffusion coefficients could not be examined. They also did not examine diffusion in the streamwise direction. The current work was conducted in an apparatus nearly identical to that used by Hsiau \& Hunt (1993a). However, the emphasis of this work is on local measurements of the velocity fluctuations in the transverse and streamwise directions and the evaluation of the diffusion coefficients in different regimes of a flow.

Besides the work by Hsiau \& Hunt (1993a), several other studies have also examined flow in a vertical channel. Examples of experiments include Nedderman \& Laohakul (1980), Savage (1979) and Takahashi \& Yanai (1973). Theoretical studies include those 


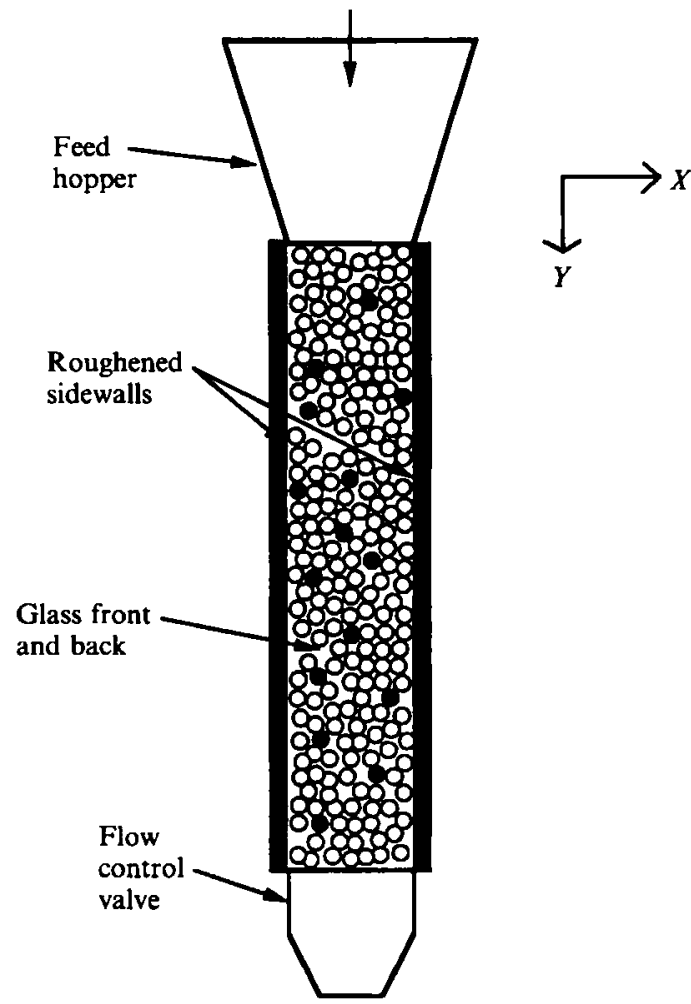

FigURE 1. Sketch of the vertical channel facility.

$\begin{array}{ccccc}\text { Expt no. } & \text { Particle type } & \begin{array}{c}\text { Exit width } \\ (\mathrm{cm})\end{array} & \begin{array}{c}\text { Mean speed } \\ \left(\mathrm{cm} \mathrm{s}^{-1}\right)\end{array} & \begin{array}{c}\text { Flow rate } \\ \left(\mathrm{kg} \mathrm{s}^{-1}\right)\end{array} \\ 1 & \text { A } & 1.0 & 5.8 & 0.091 \\ 2 & \text { A } & 1.3 & 9.5 & 0.163 \\ 3 & \text { A } & 1.5 & 12.2 & 0.233 \\ 4 & \text { B } & 1.0 & 6.7 & 0.100 \\ 5 & \text { B } & 1.3 & 10.5 & 0.175 \\ 6 & \text { B } & 1.5 & 13.9 & 0.256 \\ 7 & \text { C } & 1.0 & 6.4 & 0.098 \\ 8 & \text { C } & 1.3 & 8.8 & 0.154 \\ 9 & \text { C } & 1.5 & 11.4 & 0.217\end{array}$

TABLE 1. List of experiments

by Goodman \& Cowin (1971), Nunziato, Passman \& Thomas (1980), Savage (1979), Richman \& Marciniec (1991) and Hui \& Haff (1986). These studies are useful in evaluating and comparing the measured profiles in the present work. Most of the constitutive models currently available for the treatment of granular material flows are based either on modified plasticity models or on models derived from dense-gas kinetic theory. The plasticity models are better suited to describe behaviour in the high-density slow-deformation (quasi-static) regimes and do not account for motion at the microscopic scale of individual particles. In contrast, the models based on kinetic theory are more appropriate for flows in the 'rapid granular flow regime', characterized by binary collisions and rapid deformation. In the experiments discussed in this work, 

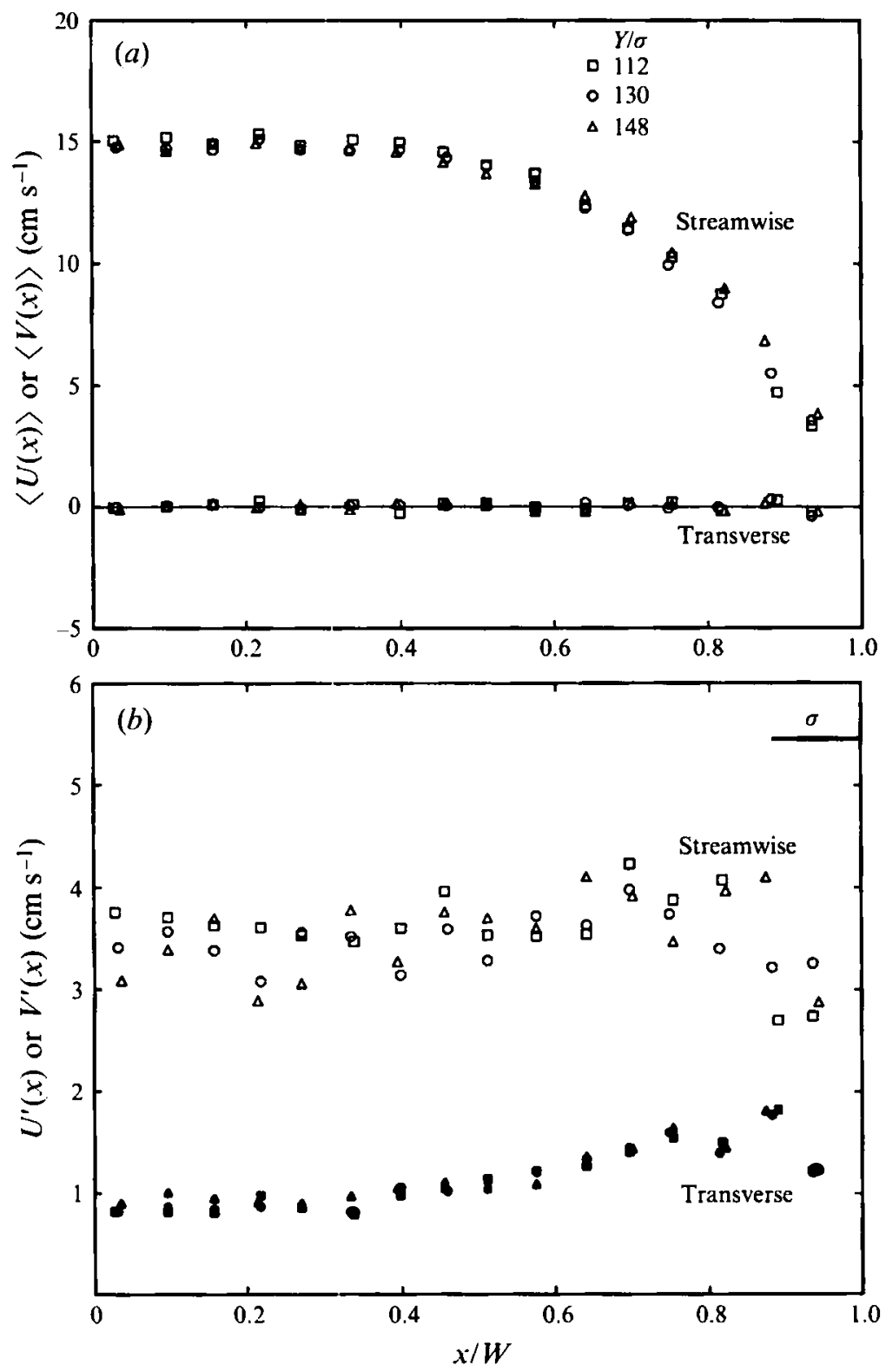

Figure 2. (a) The mean and $(b)$ the fluctuation velocity profiles at three axial positions for Expt $3 . W=2.5 \mathrm{~cm}$.

the flows are of high bulk density and visual observations indicated the presence of long-duration frictional contacts between particles. Hence, these flows belong to an intermediate regime where both frictional and collisional-translational interactions play significant roles. Johnson \& Jackson (1987) proposed constitutive relations for such flow regimes by assuming the total stress to be a simple sum of the frictional and collisional-translational contributions. 


\section{Experimental apparatus and instrumentation}

The vertical chute facility is shown in figure 1 . The chute is $100 \mathrm{~cm}$ high, $2.18 \mathrm{~cm}$ deep and $5 \mathrm{~cm}$ wide. The particles used have a nominal diameter of $3 \mathrm{~mm}$. An upper hopper feeds the particles into the test section. A flow control valve with a variable exit width controls the material flow rate. The material flow rate is measured by collecting the material exiting the test section over a specified period of time and weighing the sample. Polished tempered-glass plates were used for the front and back surfaces to minimize friction at these surfaces and to create a two-dimensional flow. The chute was dismantled and cleaned after every three runs in order to minimize the effects of dirt and static charge. As shown in Hsiau \& Hunt (1993a), the surface conditions at the sidewalls have a significant influence on the velocity and fluctuation velocity profiles. For comparison of velocity profiles, two different sidewall conditions were used: polished glass, and walls roughened by adhering $3 \mathrm{~mm}$ diameter glass spheres to the surface in a regular, approximately close-packed, hexagonal pattern.

The technique used to measure velocities was based on the work of Taylor \& Hunt (1993) and incorporated the use of imaging methods. In all cases, the flow was seeded with a small proportion of black tracer particles ( $2 \%$ by weight). Each flow was filmed using a commercial video recorder. Subsequently, sets of image sequences were digitized and stored using a frame-grabber board mounted in a personal computer. The stored digitized images contained pixel values on a grey scale of 0 to 255 ( $8 \mathrm{bit}$ ), with 0 representing black and 255 white. Each of these stored images was then processed to remove any shadows and to enhance the contrast between the black tracer particles and the non-tracer background. Then each tracer particle in the first image in a sequence was identified, along with its centroid. Subsequently the shift in a tracer particle's position between two consecutive images was determined using an autocorrelation process (Taylor \& Hunt 1993). In this autocorrelation process, a small window is drawn, in the first image, around the identified tracer particle. This window is then overlaid on the next image, but is shifted in order to account for the particle motion between the two images. The corresponding pixel values for the window, and the underlying portion of the second image are multiplied, and the resultant values summed over all pixel locations to obtain an autocorrelation value appropriate to the amount of shift applied. This process is repeated for a range of anticipated particle displacements. The shift producing the maximum autocorrelation value is assumed to represent the actual displacement of the particle. As a check, the displacement of a particle between two successive images could be calculated from the positions of its centroid in those images. The frames were acquired from the video tape at the rate of 30 frames per second (f.p.s.). The velocity was then calculated from the displacement and the time step. This measurement technique involved the averaging of velocities over a finite length of the chute which varied from 2 to $2.5 \mathrm{~cm}$. The flow section was divided into bins of equal width, in the transverse direction. In all the velocity profiles shown, the velocity and position were obtained by averaging over the velocities and positions of all the tracer particles detected within a particular bin. In all the results presented here, unless indicated, the bin width was $1.5 \mathrm{~mm}$ or half a particle diameter.

As depicted in figure 1 , the $y$-direction is the direction of mean flow, while the $x$ direction is transverse to the flow. All transverse measurements are made with reference to the axis of symmetry. For each velocity (and fluctuation velocity) profile shown, 60 sequences of images were examined, each sequence containing 108-182 images. The length of each sequence was limited by the frame-grabber board's buffer size. As a result, each data point in the velocity profile is based on an average of $250-450$ velocity 
measurements. As both the vertical and horizontal displacements could be measured, it was possible to compute the ensemble-averaged streamwise velocity $\langle V(x)\rangle$ and the ensemble-averaged transverse velocity $\langle U(x)\rangle$. The latter in all cases was very close to zero, as is expected in this flow where the mean direction of motion is vertical. The ensemble-averaged streamwise fluctuation velocities were computed as $V^{\prime}(x)=$ $\left\langle(V(x)-\langle V(x)\rangle)^{2}\right\rangle^{1 / 2}$ and the ensemble-averaged transverse fluctuation velocities as $U^{\prime}(x)=\left\langle U^{2}(x)\right\rangle^{1 / 2}$. Errors in the fluctuation velocities are less than $4 \%$ for the streamwise components and $7 \%$ for the transverse components. These errors primarily result from the uncertainty in determining the centroid of the particles.

To make the diffusion measurements, specific tracer particles were tracked for reasonable durations so that the diffusive displacements could be computed as a function of time. The actual calculations are explained in $\$ 4$. For each particle, its position in each frame of a sequence was identified and stored. In this way, the displacement-time histories for a significant number of tracer particles were accumulated. For each shear rate condition, at least 40 sequences of images, each consisting of 182 images were examined to extract the tracer trajectory data. The number of particle histories used in the diffusion calculations varied from 250 to 700 . Frames were grabbed from the video tape at the rate of $30 \mathrm{f}$.p.s., the same rate as for the velocity measurements.

All the experiments were performed using spherical glass beads with an average diameter of $3 \mathrm{~mm}$. Three different types of beads were used: clear well-rounded highquality beads with a standard deviation of $2.13 \%$ of the mean particle diameter (Type A); glass beads dyed white with an acrylic dye to alter the surface frictional properties (Type B); and clear low-quality glass beads with a diametric standard deviation of $3.34 \%$ (Type C). The dyed beads had a standard deviation of $2.88 \%$ of the mean particle diameter. The average static angle of repose was $28^{\circ}$ for particles of Type A, $31.5^{\circ}$ for particles of Type B and $28.5^{\circ}$ for those of Type C.

In order to examine diffusive behaviour, three flows with the roughened sidewalls were examined for each particle type. Table 1 lists this set of experiments, including the mean speed of each flow. Though the front and back surfaces were cleaned and polished, some frictional interaction may be expected at these walls. Also, the presence of the front and back glass walls is likely to induce a 'layered' flow structure parallel to the front wall. Liu, Kalos \& Chester (1974) performed Monte Carlo simulations for a 'classical hard sphere' system in a vertical channel and observed that layering occurred parallel to the channel walls, with the density at the walls being higher than that in the bulk. Louge (1994) observed similar density oscillations in a rapid granular flow simulation using a periodic Couette geometry that was bounded by a flat frictional wall at the bottom. In the current experiments, any shear at the front and back walls is likely to dilate the flow at those surfaces. Furthermore, the plane of shearing is perpendicular to the front wall (unlike the configuration examined by Louge 1994) and density variations caused by the presence of the front and back walls are likely to be affected by the shear-induced dilation of the bulk. Hence, it is difficult to assess the net effect of the front and back surfaces on the diffusion coefficients. However, for the particles of Type A, it was possible to observe the movement of tracer particles within the bed and to estimate the effect of the glass walls on the average velocities. The measurements indicated that the velocity within the bed was slightly higher than at the wall. The difference increased with flow rate and was about $4 \%$ for the smallest valve opening and $8-9 \%$ for the largest valve opening. This difference led to large values of the solid fractions, which were calculated using average velocities measured at the surface. Calculated values for the average solid fraction varied from 0.55 to 0.7 . While 
it was visually obvious in the case of the roughened sidewalls that the solid fraction increased from the edges towards the centre of the flow, no efforts were made to actually measure the density profile since the variations would be within the uncertainty of the measurement techniques.

\section{Velocity profiles: observations and discussion}

A significant portion of this work deals with the determination of average and fluctuating velocity profiles for both the streamwise and transverse directions. In all cases considered, the flow was fully developed and steady over the section of interest. As the flow was symmetric about the central axis, only the right-hand profile is displayed in all the figures. Unless indicated, all the figures are for the 'rough walls' condition.

Figure 2 indicates a typical example of the ensemble-averaged local velocities measured at three axial stations along the flow. This set of data is for experiment 3 . As can be seen, both the mean and the fluctuation velocity profiles are consistent for all three stations. Figure 3 shows velocity profiles for the same flow as figure 2 except that the measurements are made over three distinct averaging intervals that were two minutes apart. All the profiles are consistent, indicating that both mean and fluctuating velocities are steady.

The effect of wall conditions on the velocity profiles is shown in figure 4 . Both the cases have identical exit valve widths of $1.3 \mathrm{~cm}$. For the polished glass sidewalls, the shear layer is only 1 particle diameter thick, and a plug flow occurs across most of the flow section. The velocity at the wall, in this case, is only about $4 \%$ less than the velocity in the bulk. In contrast, for the roughened walls, the shear layer is about 6 or 7 particle diameters thick. Also, the fluctuation velocity values are higher in the case of the rough walls than in the case of the smooth glass walls. For the roughened walls, shear work at the wall is converted into the random kinetic energy of the individual particles, which is conducted into the bulk. Furthermore, visual observations indicate that for the roughened sidewalls, there is a definite decrease in solid fraction from the centre towards the walls. However, for the smooth sidewalls, the absence of any shear across most of the flow leads to a nearly uniform solid fraction across the section. In the latter case, the material seems to approach a close-packed condition, which is reflected in the low fluctuation velocity values. Hsiau \& Hunt (1993a) showed that there was negligible transverse diffusion across the central axis for the smooth wall condition.

Figure 5 compares the velocity profiles for particles of Type $\mathrm{A}$ at three different flow rates (Expts 1-3). The profiles are similar and can be scaled by the centreline velocity. Similar scaling is observed for the other particle types considered, though there is some dispersion in the high-shear regions. Slip velocities are about $20 \%$ of the centreline velocities. The profiles for the fluctuation velocities are also similar for the three flows.

An important feature is the marked anisotropy of the fluctuation velocities, with the component in the direction of mean motion being significantly higher than the transverse component. This feature manifests itself during the diffusion process. It was noticed that the fluctuation velocity profiles are scaled reasonably well by the local mean velocity, but the significance of this feature is not clear, given the lack of knowledge of the appropriate constitutive relations that govern this particular flow situation. Also, for all cases, the transverse fluctuation velocity increases from a minimum (non-zero) value at the centre to a maximum close to the wall. However, particles in the layer immediately adjacent to the wall have a lower fluctuation velocity 
V.V.R. Natarajan, M. L. Hunt and E. D. Taylor
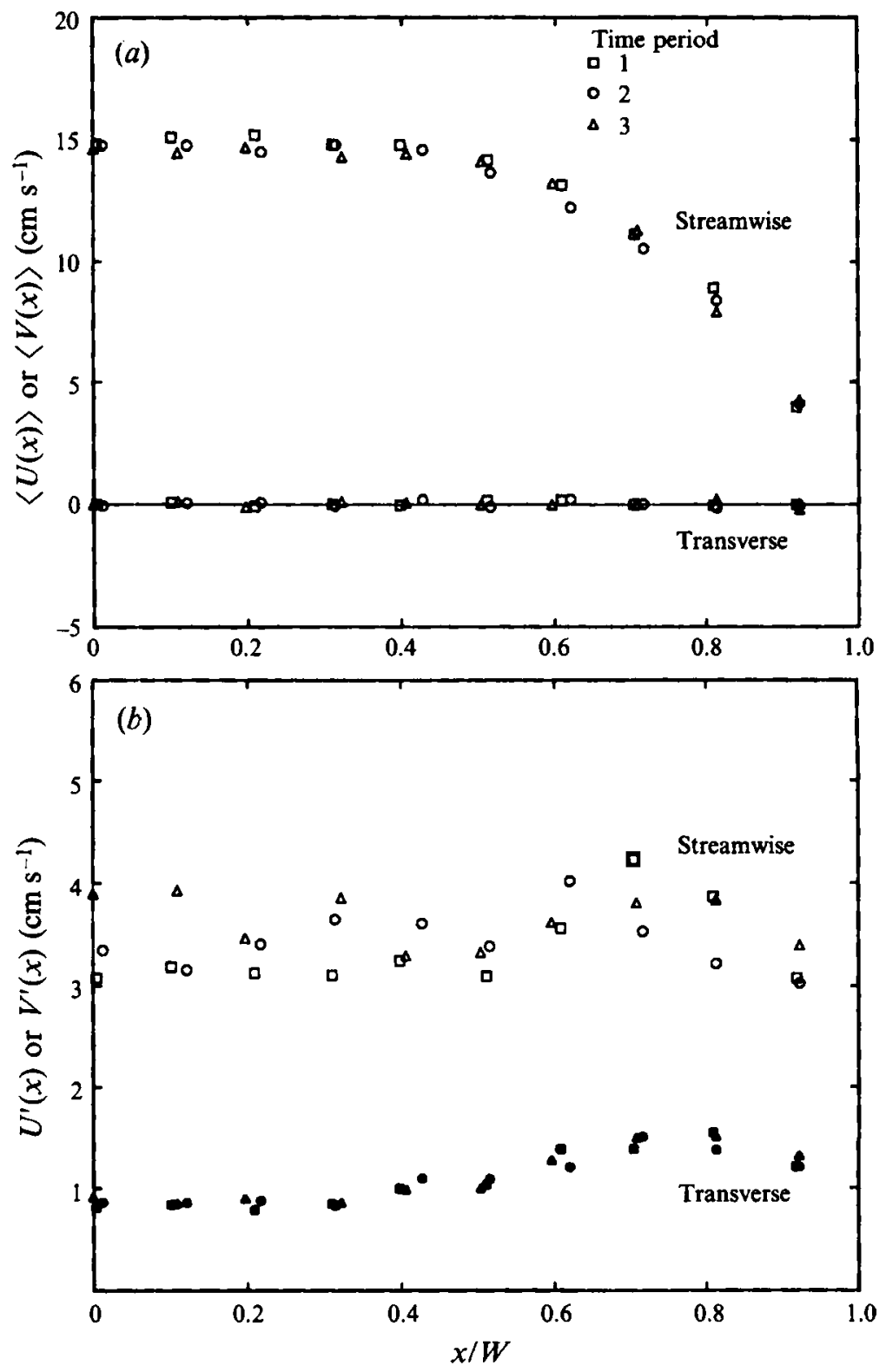

FIGURE 3. (a) The mean and $(b)$ the fluctuation velocity profiles for three distict time periods in Expt 3. $W=2.5 \mathrm{~cm}$. Bin width $=0.25 \mathrm{~cm}$.

than those centred approximately one particle diameter away from the wall. Friction between the particle surface and the wall may impart a significant rotational velocity to the particles closest to the wall. Subsequent collisions of these particles with particles further away from the wall may convert much of this rotational energy into translational energy, which manifests itself as an increase in the translational fluctuation velocity. However, a similar variation in the streamwise fluctuation velocities is not evident; variations across the channel are significantly less.

The mean velocity profiles and visual observations suggest that the flow shows a smooth transition from a uniform-velocity highly dense central region, to a relatively 

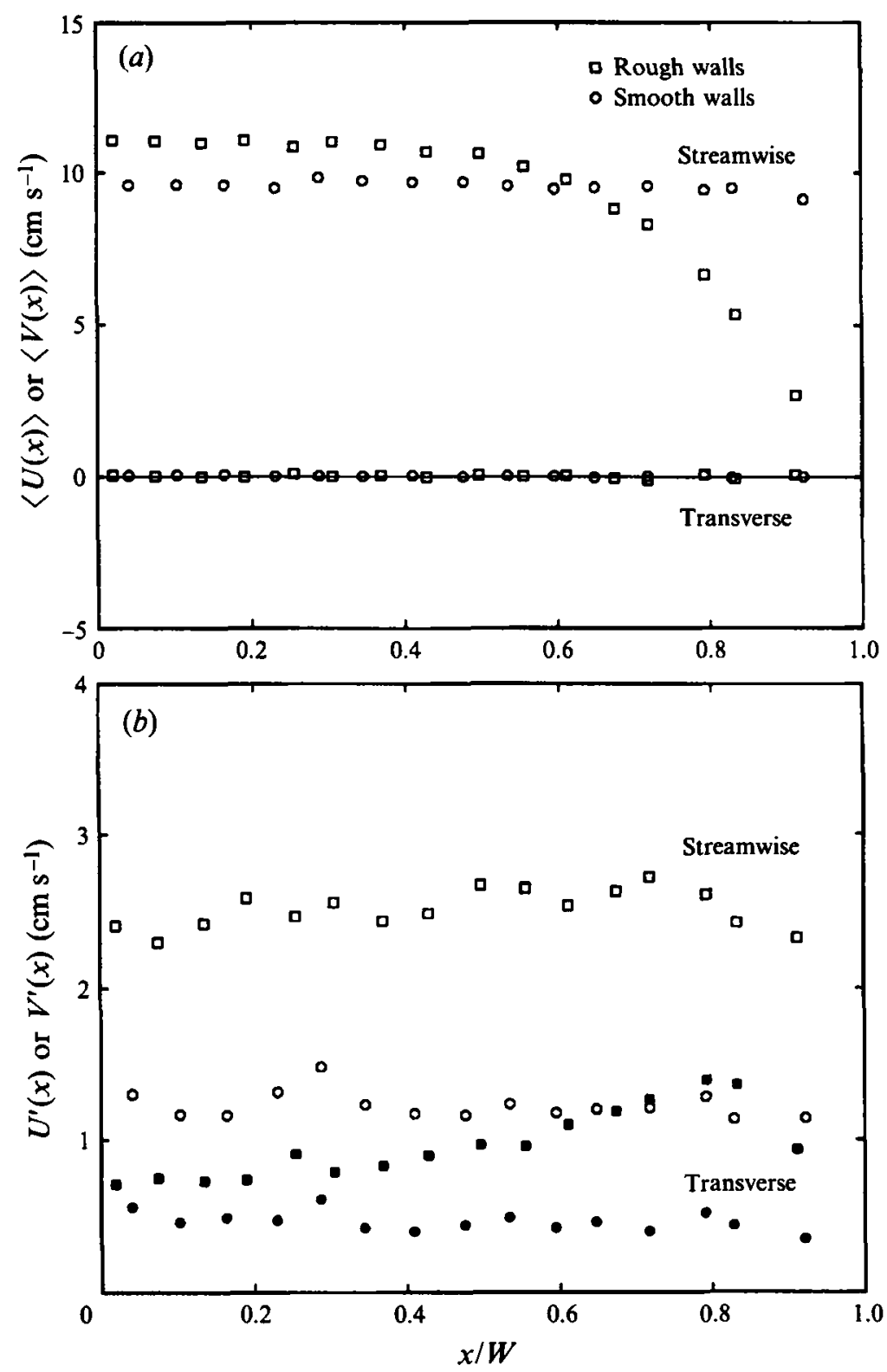

FIGURE 4. (a) The mean and (b) the fluctuation velocity profiles for smooth glass and rough walls. Exit valve width $=1.3 \mathrm{~cm} . W=2.5 \mathrm{~cm}$.

less-dense sheared regime close to the walls. From visual observations, it is evident that particles in the central region interact exclusively through semi-permanent sliding contacts at very high densities. The long-term frictional contacts dominate in the outer shear layer as well. The binary collision hypothesis, central to the development of constitutive relations based on dense-gas kinetic theory, does not appear to be applicable here.

Almost all the theoretical analyses of flow in vertical channels predict a central plug regime under certain conditions, along with a sheared regime along the walls. Hui \& Haff (1986) have used relations developed by Haff (1983) for kinetic grain flow at very 

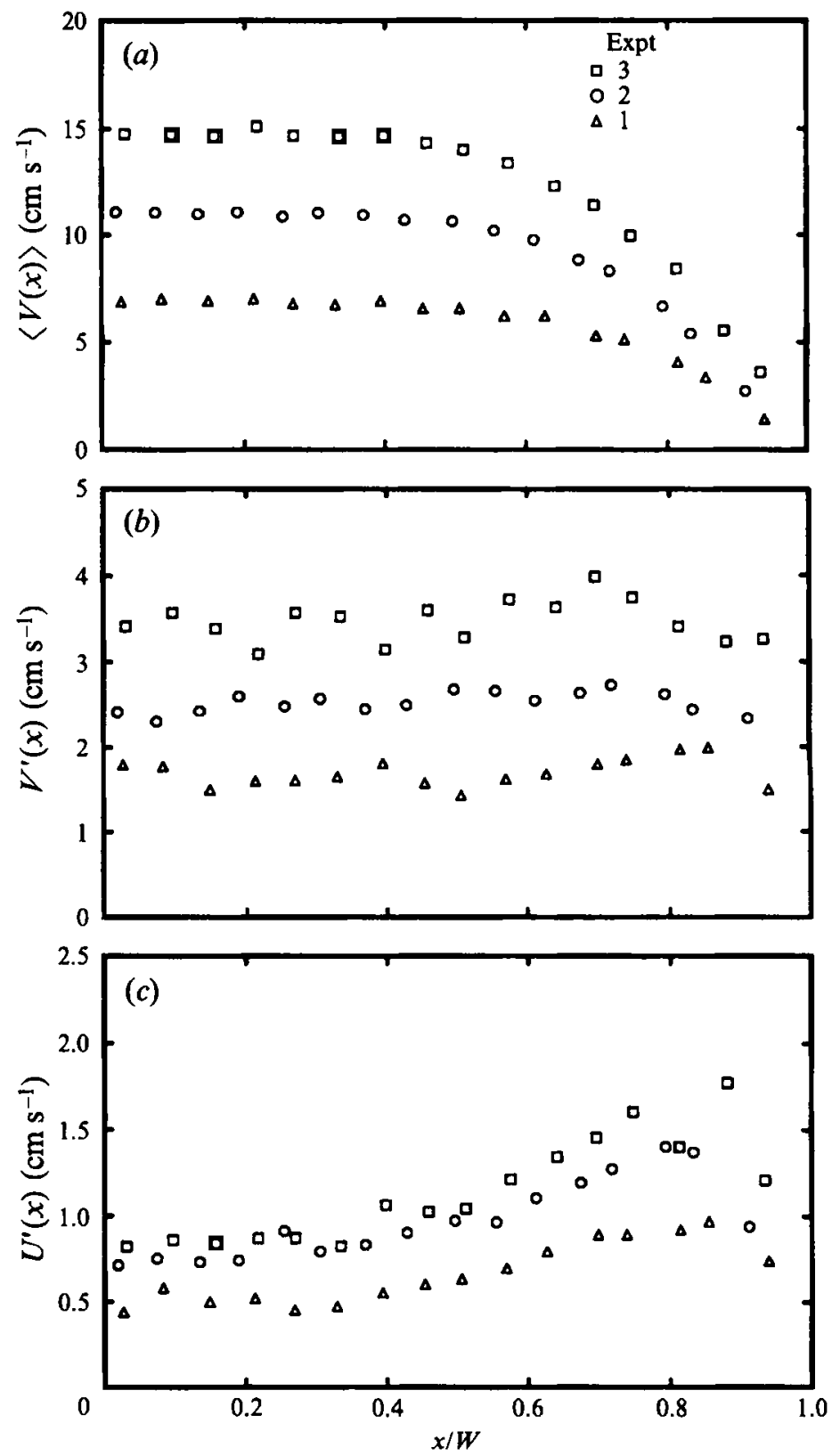

FIGURE 5. (a) The mean streamwise, (b) streamwise fluctuation and (c) transverse fluctuation velocity profiles for Expts $1-3 . W=2.5 \mathrm{~cm}$.

high concentrations to generate solutions for such flows. While their treatment was based on a binary-collision hypothesis, and did not include the effects of friction, their results for the mean velocity profiles and the 'thermal' velocity profiles have some qualitative similarities with the measured profiles. One important factor is that the analytic treatments for this geometry are based on the 'continuum hypothesis', which requires that changes in flow properties occur over lengthscales that are large relative to the particle scale. However, figure 5 shows that in the shear layer, significant changes in the mean velocity occur over distances of half a particle diameter. 

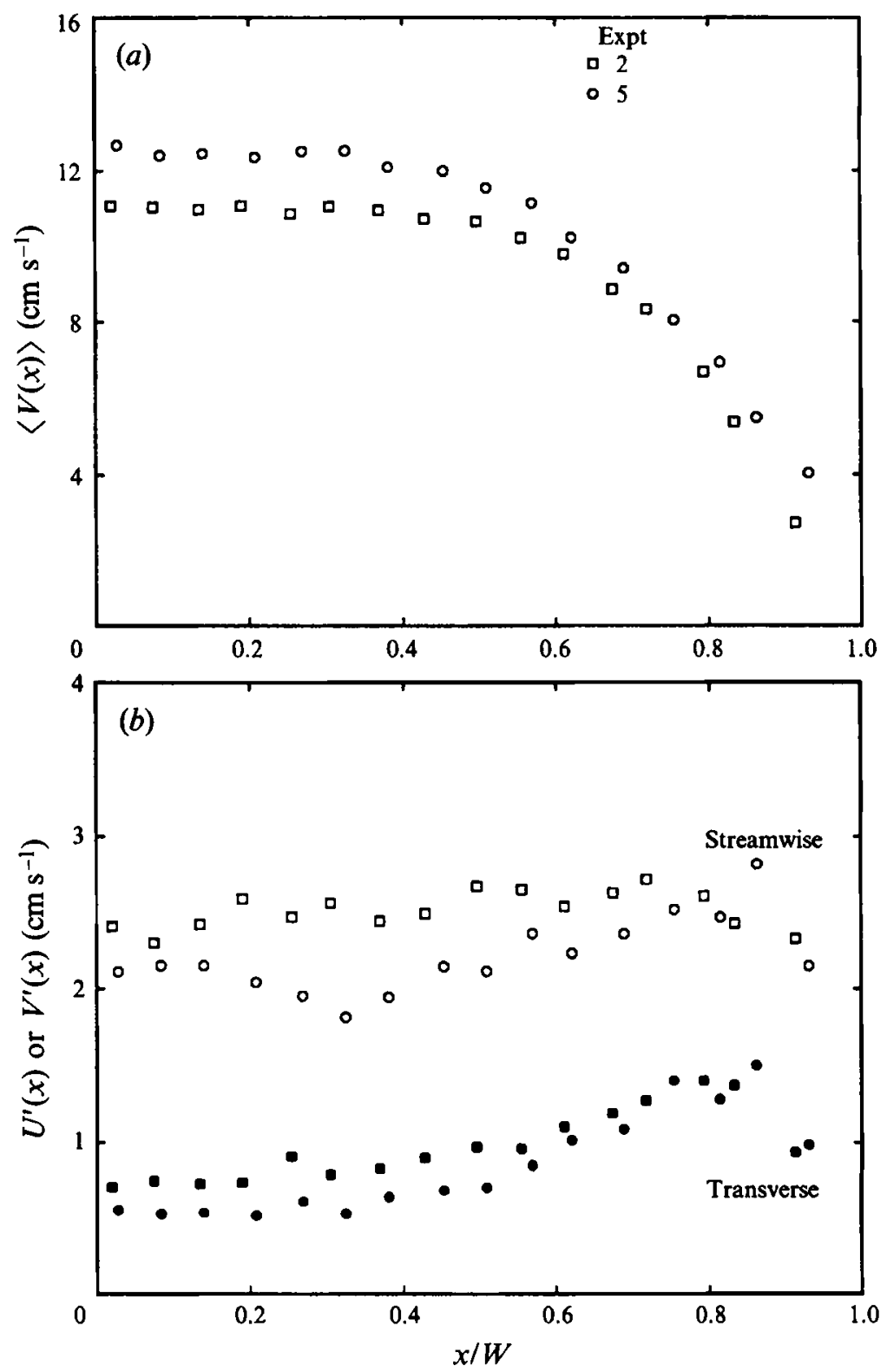

Figure 6. (a) The mean streamwise and (b) the fluctuation velocity profiles for Expts 2 (type A particles) and 5 (type B). $W=2.5 \mathrm{~cm}$.

Figure 6(a) compares the mean velocity profiles for particle types $\mathrm{A}$ and $\mathrm{B}$. The results presented are for experiments 2 and 5 . For all exit openings, it was observed that the corresponding mean flow speed was higher for the dyed particles than for the clear particles. The reason for this phenomenon is not clear, though it might be due to the exit hopper condition (Nguyen, Brennen \& Sabersky 1979; Brennen \& Pearce 1978). Figure $6(b)$ compares the fluctuation velocity profiles for the same cases as figure $6(a)$. The dyed particles have lower fluctuation velocities across most of the bulk, indicating a significant damping of the translational fluctuation velocities due to friction.

Normalized fluctuation velocity distributions were calculated from the velocity data 
accumulated from each data point. Figure 7 compares the normalized transverse fluctuation velocity distributions, at three representative transverse locations, for the rough and smooth wall conditions. In these figures $f(U)$ represents the fraction of the total number of velocity observations for each data point that were equal to the velocity $U$. A similar comparison between the streamwise fluctuation velocity distributions is displayed in figure 8 . The distributions for the condition of the rough wall approximate a Maxwellian distribution while those for the smooth glass wall conditions deviate substantially.

\section{Self-diffusion: review of theory}

Self-diffusion refers to the motion (displacement) of particles in a monodisperse system, which cannot be accounted for by the mean motion of the material. It arises as a consequence of the existence of the random fluctuation velocity components. Savage \& Dai (1993) and Campbell (1993) have considered the diffusive behaviour of granular systems in a manner analogous to the diffusive behaviour of suspended particles undergoing Brownian motion in a liquid (Einstein 1956). Under these circumstances, were a system to be simply diffusive, the diffusive displacements or the mean-square particle displacement due to the random velocity components can be related to the coefficient of self-diffusion $\mathscr{D}$ by the kinematic expression:

$$
\lim _{t \rightarrow \infty}\left\langle|R|^{2}\right\rangle=2 \mathscr{D} t,
$$

where $R$ is the diffusive displacement and $t$ is the time.

In the case of a granular flow, the fluctuation velocities may be anisotropic, as noted in $\S 3$. Hence, under anistropic circumstances, it is more appropriate to define a diffusion coefficient tensor $D_{i j}$ as

$$
\lim _{t \rightarrow \infty}\left\langle\Delta x_{i} \Delta x_{j}\right\rangle=2 D_{i j} t,
$$

where $\Delta x_{i}$ is the diffusive displacement in direction ' $i$ '. It must be noted, that for very short timescales, the root-mean-square diffusive displacements vary linearly with time.

Hsiau \& Hunt (1993b) and Savage \& Dai (1993) used kinetic theory arguments to arrive independently at the following expression for the self-diffusion coefficient:

$$
D_{i i}=\frac{\sigma(\pi T)^{1 / 2}}{8\left(1+e_{p}\right) v g_{0}(v)} .
$$

Here, $\sigma$ is the particle diameter, $T$ is the granular temperature, $e_{p}$ is the coefficient of restitution of the particle, $\nu$ is the solid fraction and $g_{0}(\nu)$ is the radial distribution function. In the case of perfectly elastic particles, equation (4.3) reduces to the classical Chapman-Enskog expression.

Each of the above equations is based on the assumption that the fluctuation velocity field is homogeneous and stationary. Equation (4.3) has the added assumption that the fluctuation velocity field is isotropic. The applicability of equation (4.2) for the determination of the diffusion tensor in simple shear flows is convenient since for a simple unbounded granular shear flow, with a uniform shear rate, the generation of granular temperature through shear work is balanced by its dissipation through collisions. Therefore, even if the mean velocity field is inhomogeneous, the fluctuation 

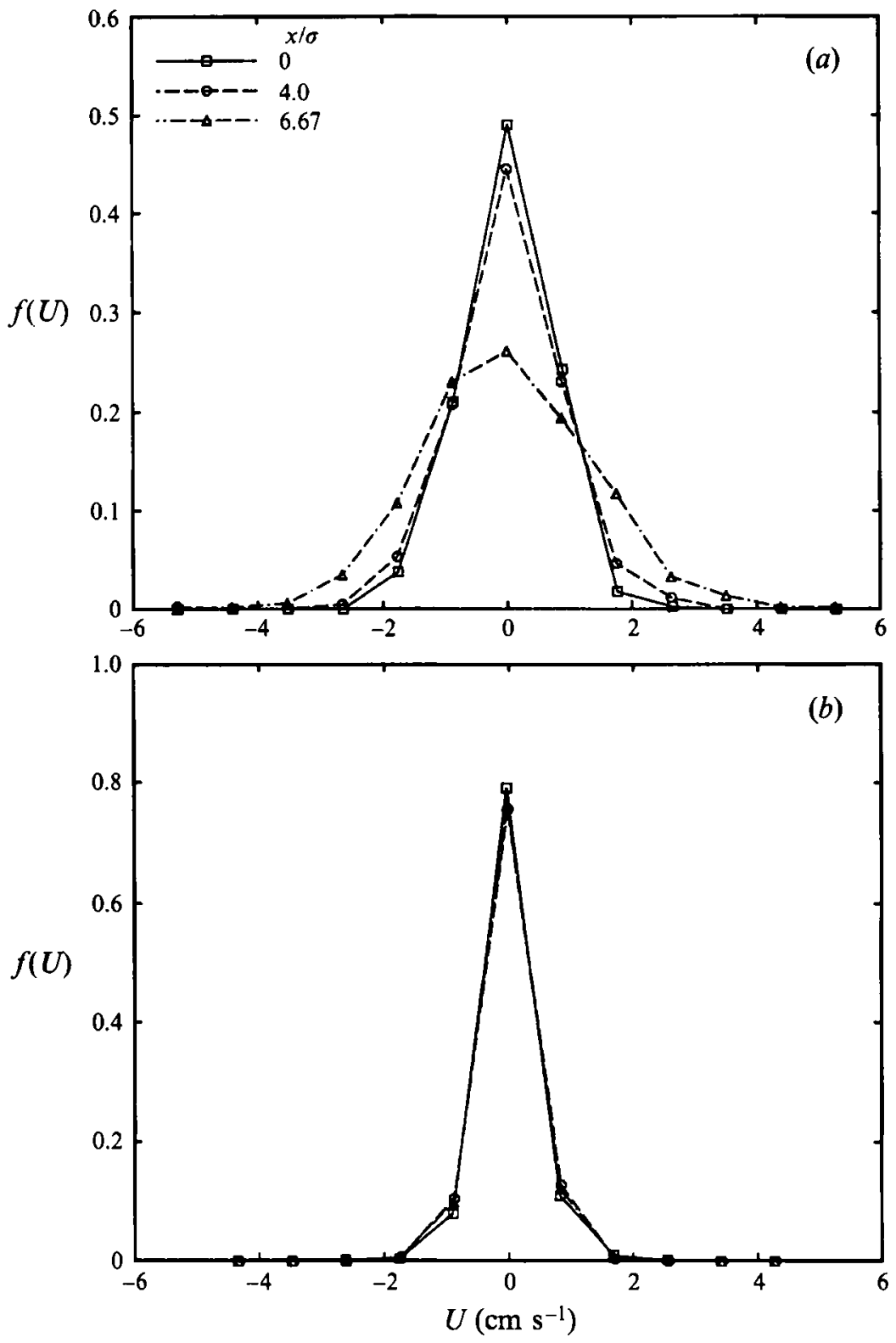

FIGURE 7. Distribution of transverse fluctuation velocities for (a) rough and $(b)$ smooth walls. Exit valve width $=1.3 \mathrm{~cm}$.

velocity field is homogeneous and the granular temperature is only a property of the strain rate and the material. However, such a uniform fluctuation velocity field is rarely encountered in experiments where the boundaries serve as sources or sinks for the random kinetic energy. The presence of boundaries leads to gradients in the fluctuation velocity, as noted in the previous section.

In order to make realistic comparisons between the shear rates, fluctuation velocities and diffusion coefficients, the flow section in each experiment was divided into three regimes. These were the central high-density uniform-flow regime, the moderate-shear regime close to the walls, and the intermediate low-shear regime connecting these two 

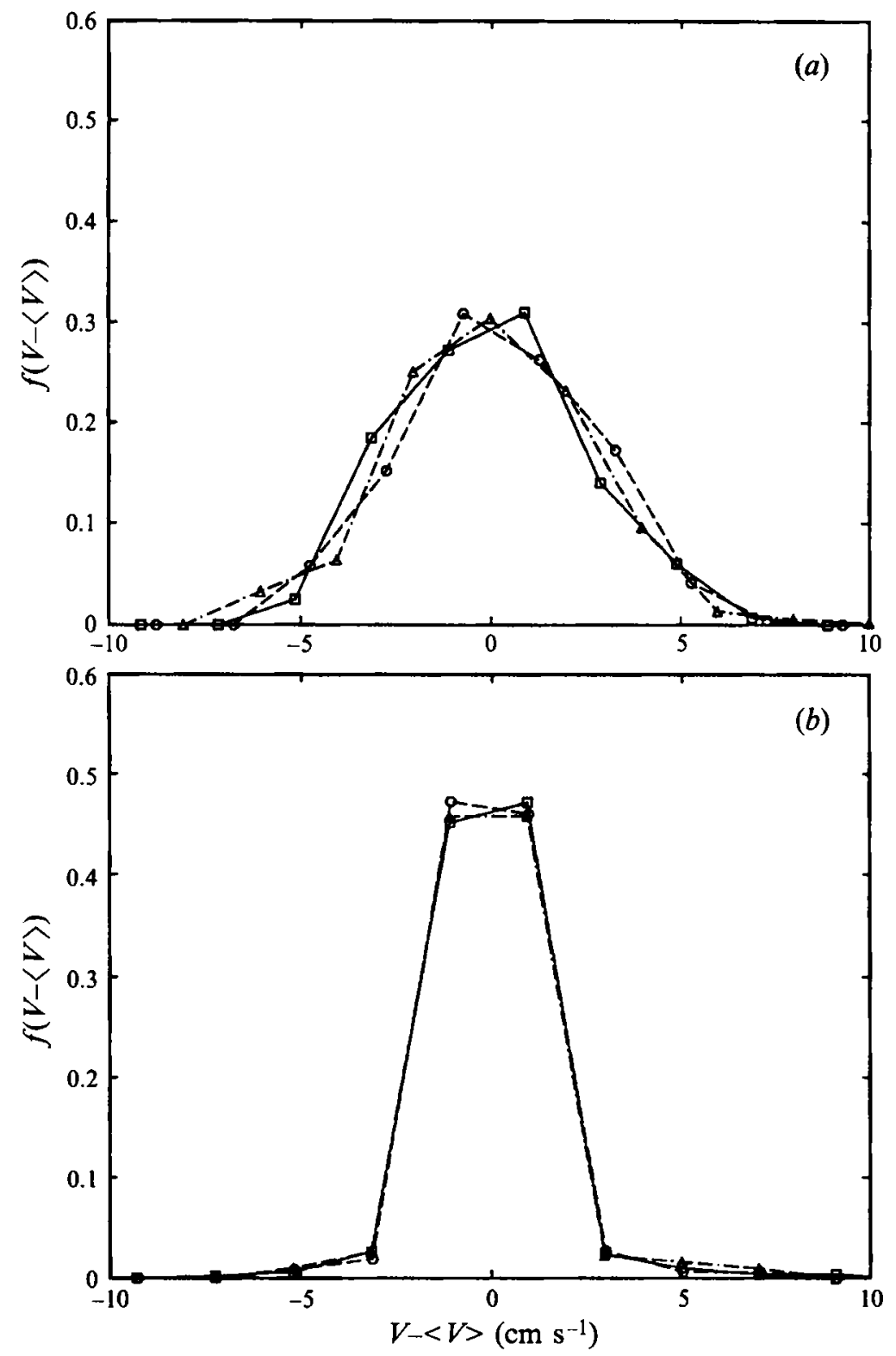

FIGURE 8. Distribution of streamwise fluctuation velocities for $(a)$ rough and $(b)$ smooth walls. Exit valve width $=1.3 \mathrm{~cm}$. Symbols as figure 7.

regimes. The latter regime is referred to as the 'low-shear transition regime' in subsequent discussions. Within each regime, the streamwise and transverse fluctuation velocities were relatively uniform with standard deviations for the streamwise fluctuation velocities around $5-7 \%$ of the mean value. The standard deviations for the transverse fluctuations were the highest in the moderate-shear regime, being about $9-12 \%$ of the mean value. The deviations of the transverse fluctuations were much lower in the two inner regimes. The present work approximates each of the three regimes as a simple shear flow. Each of the sheared regimes was 2-2.5 particle diameters wide. As the averages indicated in equation (4.2) were carried out over 
ensembles of particles that were tracked at different times and initial positions in the flow, the flows were assumed to be homogeneous and steady within each regime of interest. Figures 2 and 3 indicate that these are reasonable assumptions. When tracking particles in the moderate-shear regime, particles within a distance of 1.5 diameters from the wall were not considered in order to avoid particles that bounce off the walls and any three-dimensionality effects at the corners.

A phenomenon that arises because of the mean velocity gradient is 'Taylor dispersion' (Taylor 1953, 1954). Transverse fluctuations cause a particle to be displaced transverse to the direction of mean motion to a position where the mean velocity is different from that at its initial position. This velocity difference manifests itself as an apparent diffusive displacement of the particle away from its initial position. Therefore, diffusive displacements in the direction of mean motion are composed of two components: one due to the random fluctuation velocity and the other due to the gradient in mean velocity. To determine the diffusion component due to the random fluctuations at the end of each time step, it was necessary to subtract from a particle's total diffusive displacement, the displacement contribution due to the mean velocity difference during that time step, which was done by referring to the mean velocity profile. The remaining component is referred to as the 'random' diffusion component in subsequent sections.

The diffusion coefficient based on kinetic theory arguments indicates that diffusion increases with an increase in granular temperature and with the diameter of the particles. Furthermore, as $v g_{0}(v)$ is an increasing function of the solid fraction, diffusion decreases with an increase in the solid fraction. Although kinetic theory arguments may not be appropriate at high solid fractions, it is expected that the diffusion coefficients at high solid fractions would have a similar dependence on the fluctuation velocities, the particle diameter and the solid fraction, as long as a rigid close-packed state is not reached. Therefore, a non-dimensional parameter $D 1_{i}$ :

$$
D 1_{i}=\frac{D_{i i}}{\sigma U_{i}^{\prime}},
$$

is defined to examine the dependence of the diffusion coefficient on the fluctuation velocity $U_{i}^{\prime}$ and the particle diameter. However, it is impossible in this work to quantify the density dependence because the actual density profiles were not measured.

Another non-dimensional parameter used by Bridgewater (1980), Campbell (1993), and Savage \& Dai (1993) is $D 2_{i}$ :

where $\gamma$ is the strain rate.

$$
D 2_{i}=\frac{D_{i i}}{\sigma^{2} \gamma},
$$

In the case of simple uniform-shear flows, at moderate solid fractions, the granular temperature depends only on material properties and the strain rate; hence $D 1$ or $D 2$ could be used interchangeably. In this work, both coefficients were considered since the functional dependence of the fluctuation velocities on the strain rates is not known.

\section{Self-diffusion: observations and discussion}

Figure 9 shows the variation of $\langle\Delta x \Delta x\rangle$ with time for experiment 3 . The meansquare transverse displacements increase linearly with time in each of the three regimes. Diffusive displacements are the highest in the moderate shear regime, significantly less in the low-shear transition regime and least, but non-zero, in the central uniform-flow 


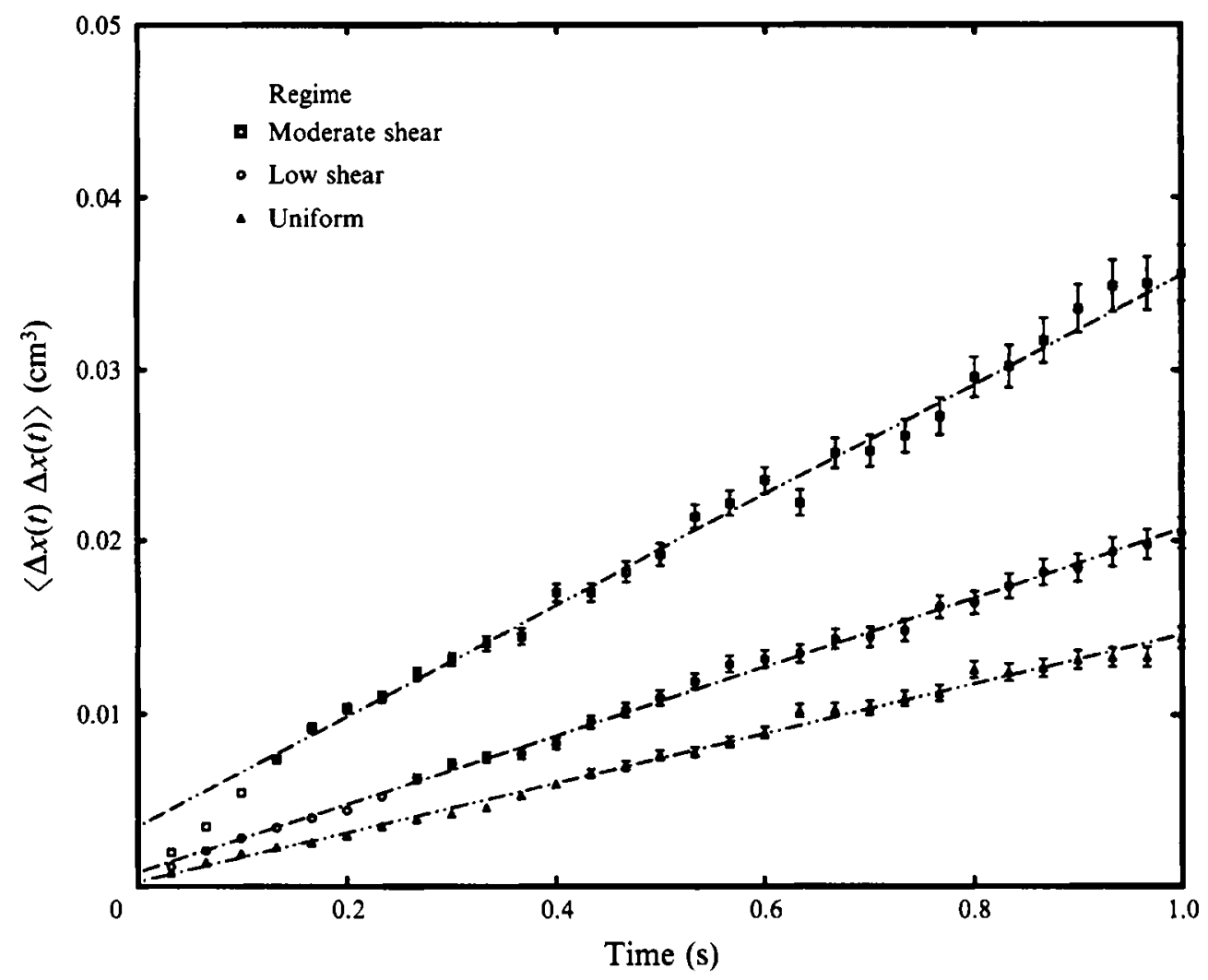

FIGURE 9. Variation of mean-square transverse diffusive displacement with time: Expt. 3.

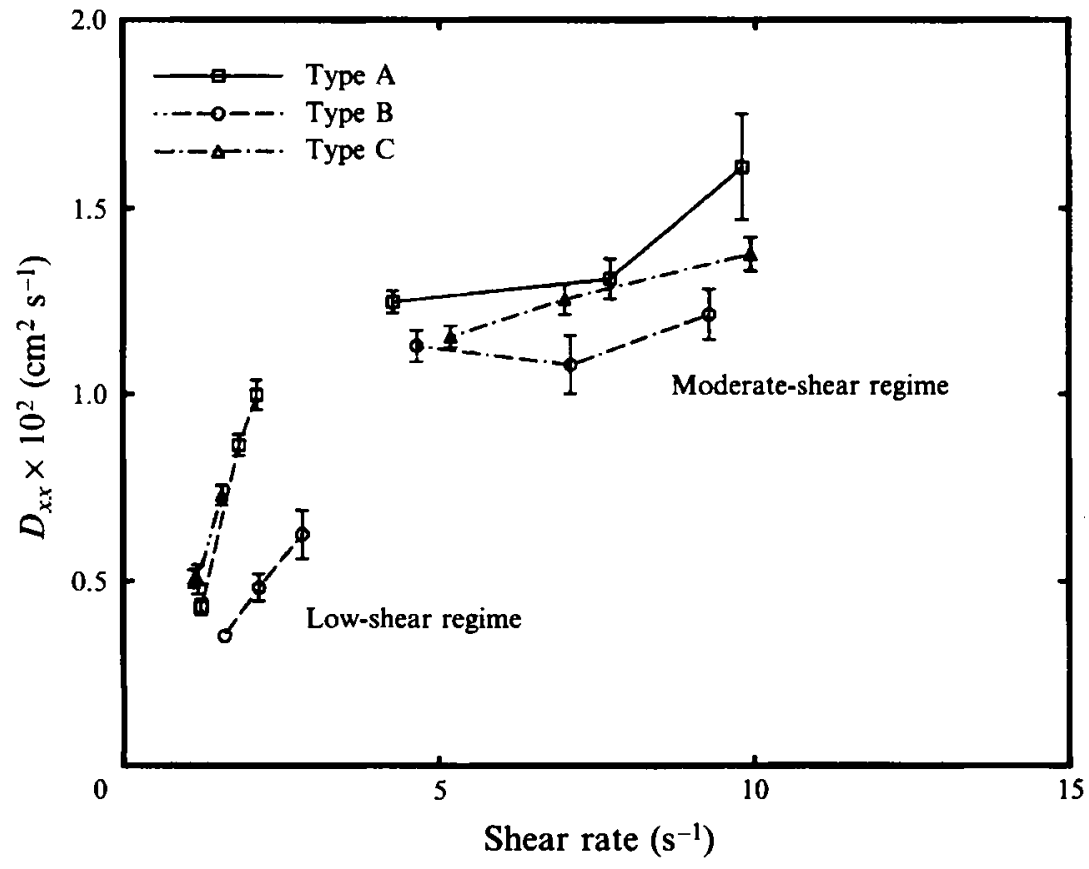

Figure 10. Variation of the coefficient of transverse diffusion $D_{x x}$ with shear rate for Expts 1-9. 

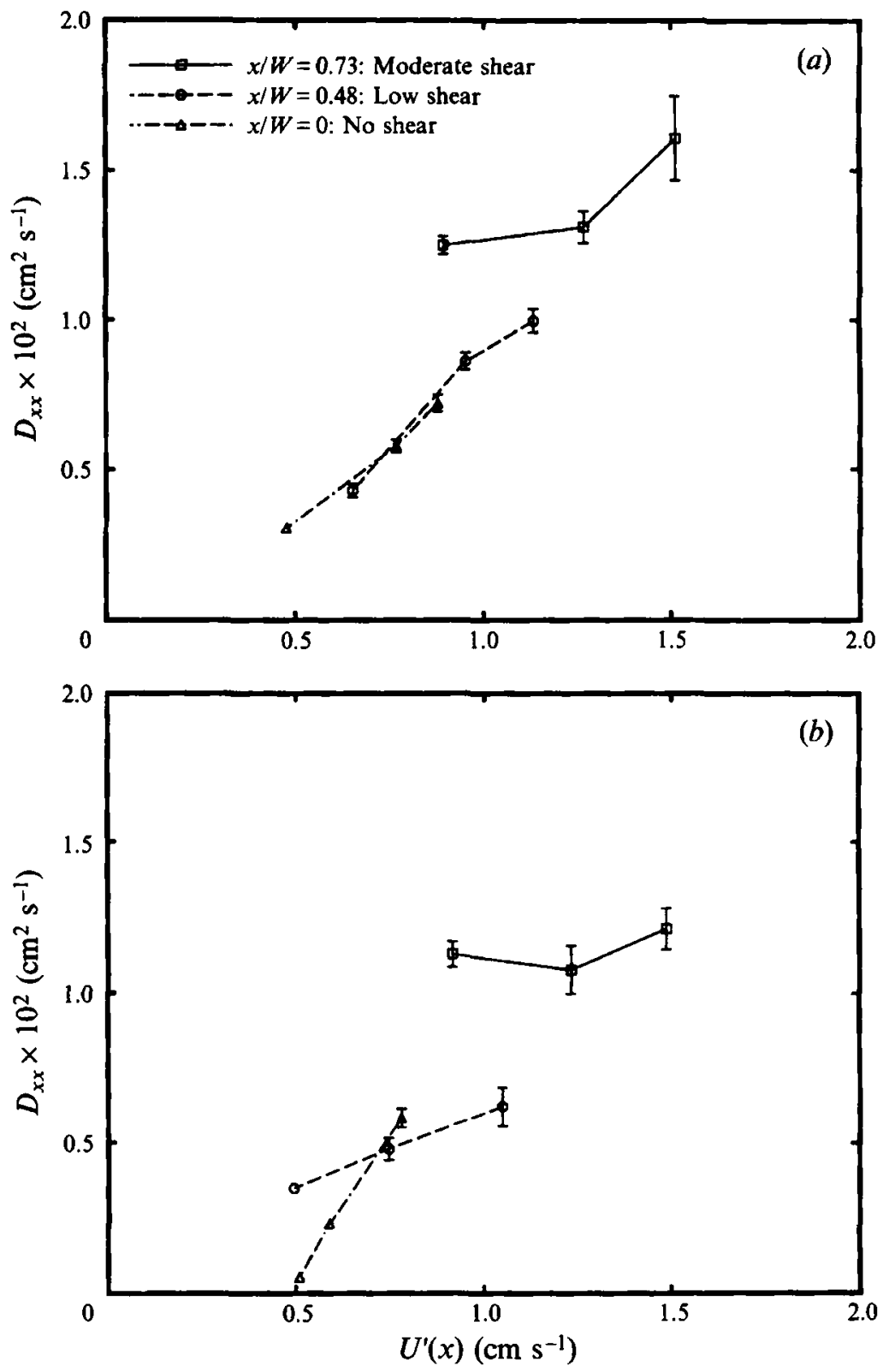

FigurE 11. Variation of the coefficient of transverse diffusion $D_{x x}$ with transverse fluctuation velocity for particle types (a) A and (b) B.

regime. This trend is consistent in all the flows examined. The error bars in the figure reflect the uncertainty in determining the centroid of a particle. The uncertainty in the slope of the best fit line ranged from $3 \%$ to $8.8 \%$.

The variation of $D_{x x}$ with shear rate for all three particle types is depicted in figure 10. For all particle types, diffusion coefficients are much higher in the moderate-shear regime than in the low-shear transition regime. However, diffusion is observed to be a much more sensitive function of shear rate in the transition regime than in the moderate-shear regime. Also, for comparable shear rates, the particles of Type B have lower values of $D_{x x}$ than those for Type A. The simulations by Dai (1993) showed a 
significant reduction in the coefficients of diffusion for rough particles. However, results for solid fractions of more than 0.5 were not presented. A feature common to all measurements in the moderate-shear regime is that the values of the self-diffusion coefficients for particles of Type $\mathrm{C}$ are comparable to values obtained for Type $\mathrm{A}$ and larger than those for Type B.

The variation of $D_{x x}$ with transverse fluctuation velocity for particles of Type $\mathrm{A}$ is shown in figure $11(a)$. Again, diffusion increases more rapidly with an increase in fluctuation velocity in the uniform and the low-shear regimes than in the moderateshear regime. Qualitatively similar results are observed in figure $11(b)$ for the case of particles of Type B.

Savage \& Dai (1993) have plotted $D 2$ as a function of the solid fraction. Although the solid fraction profiles could not be determined in these experiments, $D 1_{i}$ and $D 2_{i}$ have been plotted against the mean transverse position of each shear regime in a flow. As noted earlier, the solid fraction decreases and $D_{x x}$ increases near the walls. Figure 12 depicts the values of $D 1_{x}$ for the particles of types A and B. For particles of Type A the values of $D 1_{x}$ are very close to each other for each of the three regimes for experiments 2 and 3 . However, the values for the case of experiment 1 (the smallest exit width) deviate from this behaviour. As noted earlier, $D 1_{x}$ does not account for the solid fraction. While measurements were not made, it was visually observed that the solid fraction decreased from the centre towards the walls in all the flows. Also, theoretical analyses of such flows (Richman \& Marciniec 1991; Nunziato et al. 1980; Hui \& Haff $1986)$ indicate qualitatively similar density profiles. Such a density variation does explain the increase in $D \mathrm{I}_{x}$ with transverse position in the case of the two wider exit openings in figure 12. The reason for the deviation of the values for the case of the smallest exit width is not clear. If examined in terms of a density distribution, the results suggest that in the latter case, there is greater relative dilation in the moderateshear layer, and relatively greater compaction of the two inner layers, in comparison to the other two exit conditions. Figure 12 also shows the value of $D 1_{x}$ for the case of the particles of Type B. For the sheared regimes of experiments 5 and 6 , the values are quite consistent, though there is significant deviation in the uniform regime. The values of $D 1_{x}$ are consistently lower, in the sheared regimes, for the case of the particles of Type $\mathrm{B}$, indicating a significant reduction in diffusive behaviour due to the presence of friction.

The values of $D 2_{x}$ for the case of particles of all three types are presented in table 2. The values for the centre of the channel are not given since the shear rate here is zero and $D 2_{x}$ cannot be defined. As in the case of $D 1_{x}$, the values of $D 2_{x}$ are almost equal for the case of the sheared zones for experiments 2 and 3.

Again, results for the case of the smallest exit opening deviate from the others. Note that $D 2_{x}$ is 0.053 for the low-shear regimes in experiments 2 and 3 . This value is close to the value of 0.057 reported by Bridgewater (1980), based on the work of Scott \& Bridgewater (1976) who performed their experiments in a shear cell over shear rates in the range of $0.16 \mathrm{~s}^{-1}$ to $0.62 \mathrm{~s}^{-1}$, which is lower than but comparable to the strain rates in the low-shear transition regimes of the current set of experiments. In addition, Savage \& Dai (1993) reported that their simulations results were in good agreement with Bridgewater's (1980) results, for a solid fraction of 0.5 . They do not mention the range of strain rates examined. Measurements made from the figures of Campbell (1993) indicate the value for $D 2_{x}$ to be about 0.025 , for a solid fraction of 0.5 . Again, the range of strain rates examined is not indicated. Also, Bridgewater (1980) reported that diffusion in their experiment was isotropic when corrected for Taylor dispersion. As subsequent discussions show, this is not observed in the current set of experiments. 


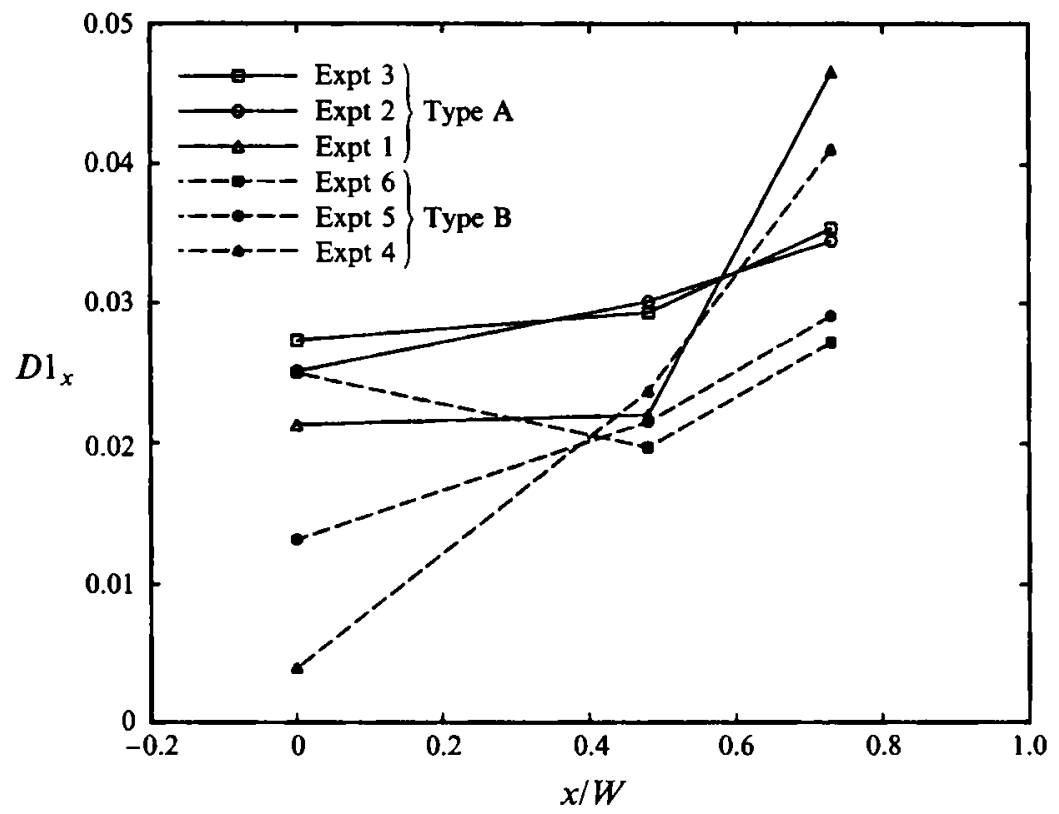

FIGURE 12. Variation of $D 1_{x}$ with position (or shear regime) for particle types $A$ and $B$. $W=2.5 \mathrm{~cm}$.

\begin{tabular}{|c|c|c|c|c|c|c|}
\hline \multirow[b]{2}{*}{ Expt } & \multicolumn{2}{|c|}{$D 2_{x}$} & \multicolumn{2}{|c|}{$D 2_{y}$} & \multicolumn{2}{|c|}{$D 2$} \\
\hline & LS & MS & LS & MS & LS & MS \\
\hline 1 & 0.039 & 0.033 & 0.511 & 0.181 & 0.549 & 0.212 \\
\hline 2 & 0.053 & 0.019 & 0.420 & 0.162 & 0.472 & 0.181 \\
\hline 3 & 0.053 & 0.018 & 0.603 & 0.194 & 0.655 & 0.212 \\
\hline 4 & 0.024 & 0.027 & 0.231 & 0.173 & 0.255 & 0.200 \\
\hline 5 & 0.020 & 0.017 & 0.310 & 0.127 & 0.335 & 0.143 \\
\hline 6 & 0.020 & 0.015 & 0.359 & 0.136 & 0.383 & 0.150 \\
\hline 7 & 0.050 & 0.025 & 0.571 & 0.160 & 0.621 & 0.185 \\
\hline 8 & 0.047 & 0.019 & 0.554 & 0.171 & 0.602 & 0.190 \\
\hline 9 & 0.052 & 0.015 & 0.660 & 0.160 & 0.712 & 0.176 \\
\hline
\end{tabular}

TABLE 2. Values of $D 2_{i}$ : LS, low shear; MS, moderate shear

The variation of $\langle\Delta y \Delta y\rangle$ with time is shown in figure 13 for experiment 3; similar results are observed for the other experiments. The figures display curves for the total diffusion, as well as for the 'random diffusion' component. As expected, Taylor dispersion contributions are largest for the moderate-shear regime, much lower for the low-shear transition regime, and non-existent in the central uniform regime, where all diffusion is due to random velocity fluctuations. As in the case of transverse diffusion, the random diffusive displacements are the highest in the moderate-shear regime, lower in the transition low-shear regime and least in the centre. This trend is true of all the flows investigated, though relative magnitudes vary. The variation of the streamwise mean-square random displacements with time is linear for all the regimes. The uncertainty in the slope of the best fit lines was between $6 \%$ and $13 \%$. The curves for total diffusion could not be fitted consistently by a single power law and were not examined further. 

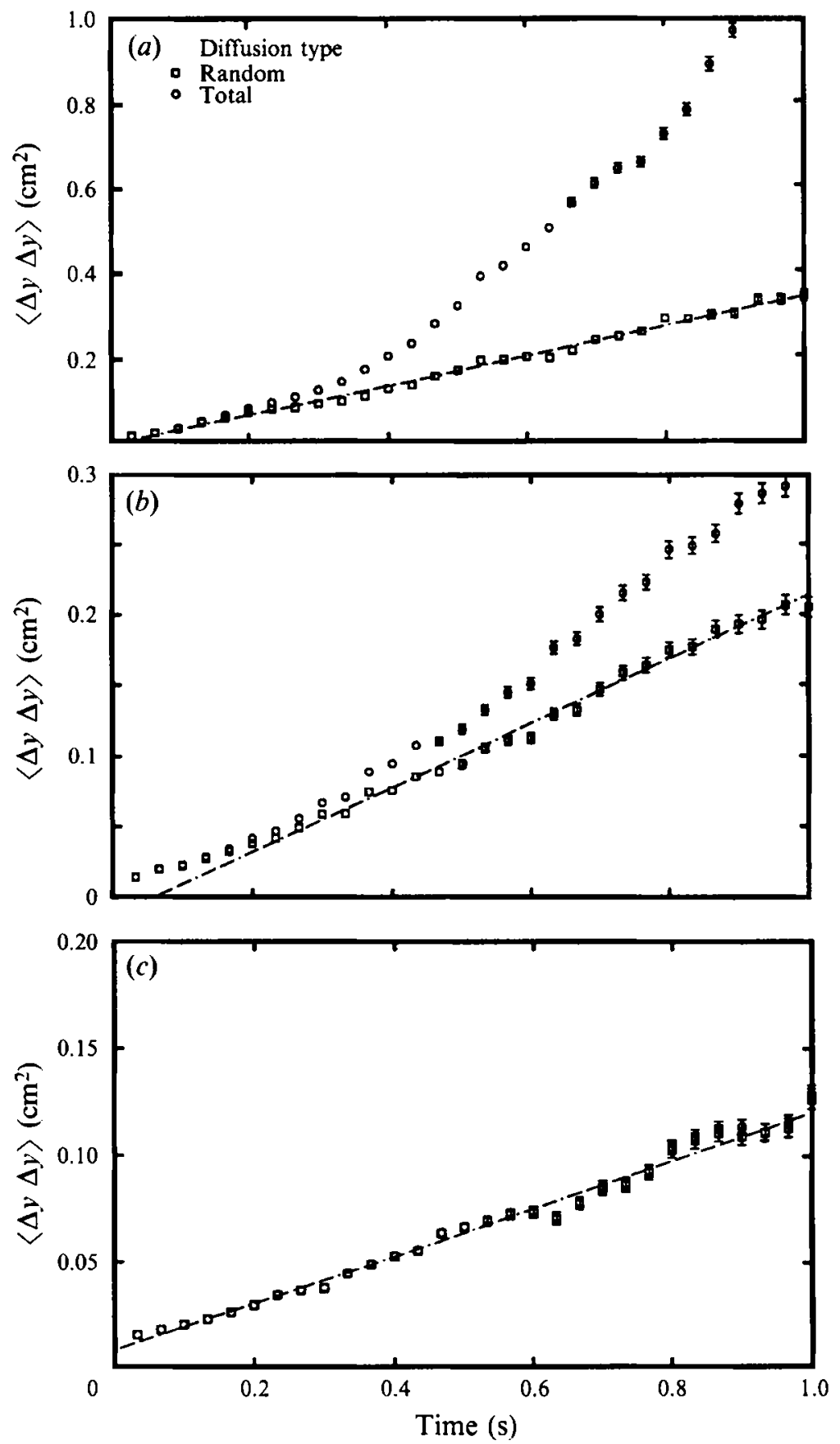

FIGURE 13. Variation of mean-square streamwise diffusive displacement with time for the (a) moderate-shear, (b) low-shear and (c) uniform regimes in Expt 3.

Figure 14 depicts the variation of $D 1_{y}$ with transverse position for the case of particles of types A and B. As for $D 1_{x}, D 1_{y}$ increases from the centre towards the highshear regime. The same arguments, based on the variation of solid fraction, may be extended in this case as well. However, the values of $D 1_{y}$ for Type $A$ are close to each other, within each regime, in all three shear regimes for all the flow rates, unlike the 


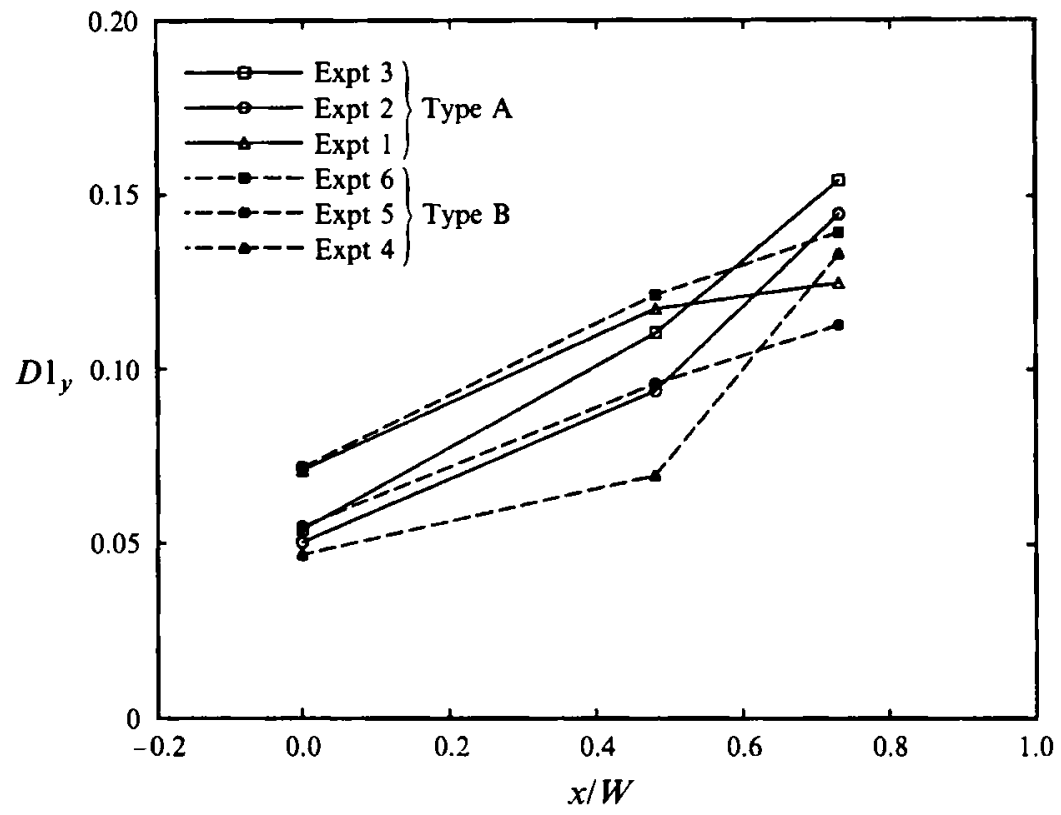

FiguRE 14. Variation of $D 1_{y}$ with position (or shear regime) for particle types $A$ and $B$. $W=2.5 \mathrm{~cm}$.

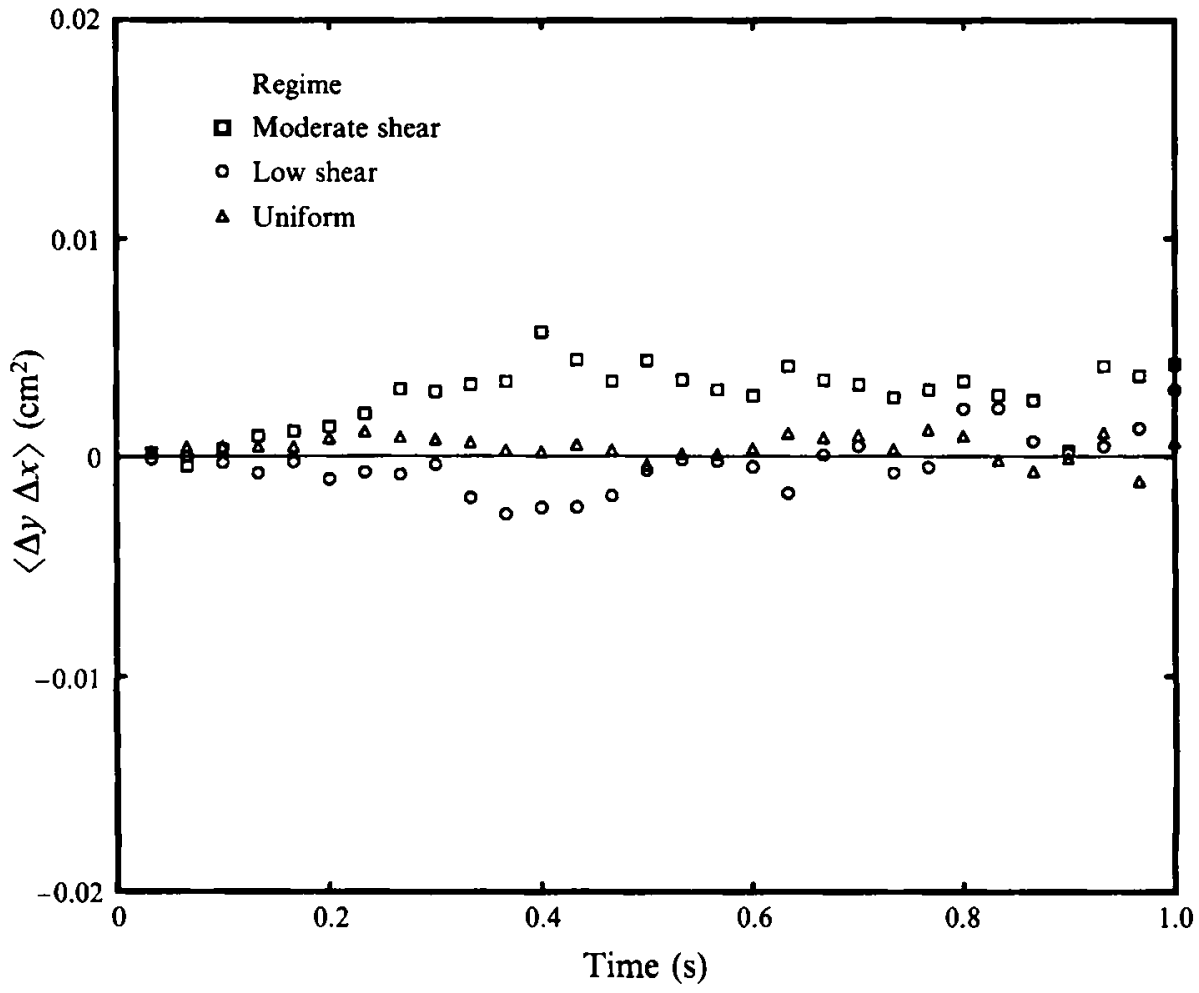

Figure 15. Variation of $\langle\Delta y \Delta x\rangle$ with time. Expt 3. 


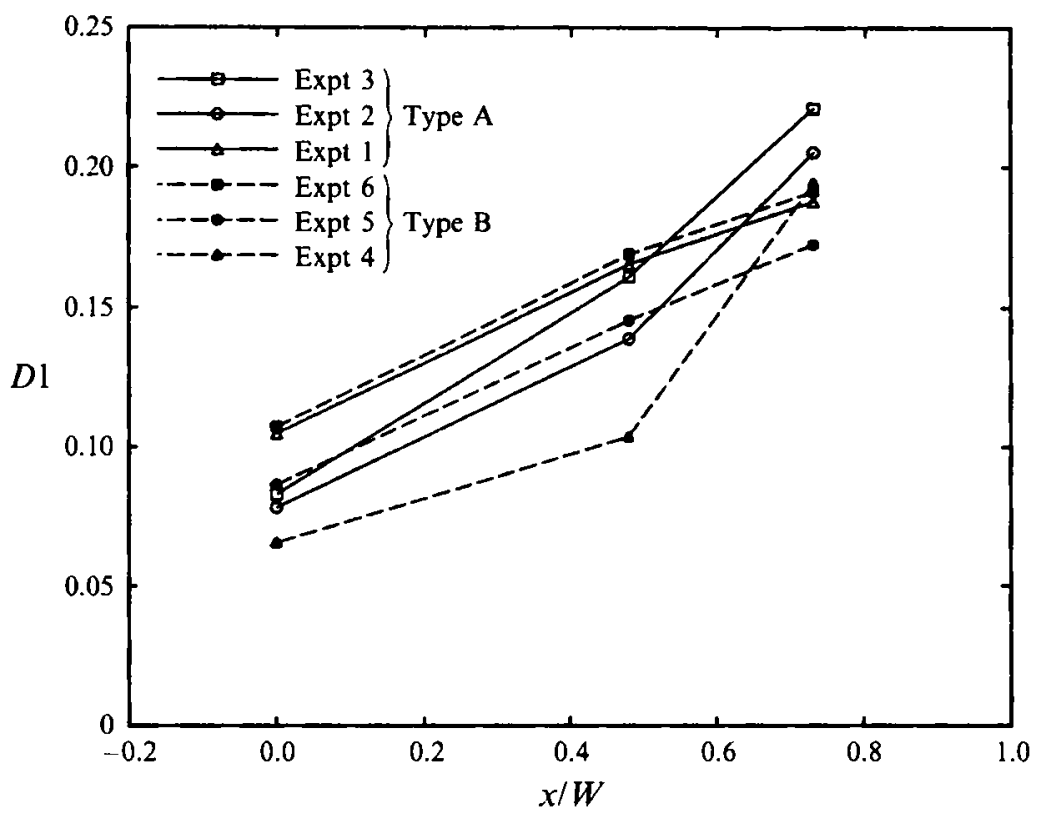

FIGURE 16. Variation of $D 1$ with position (or shear regime) for particle types A and B. $W=2.5 \mathrm{~cm}$.

results shown in figure 12. The results for particles of Type B are similar to those for the particles of Type A, though not as consistent amongst the three different flow rates. The values of $D 2_{y}$ for the sheared regimes are listed in table 2. In comparison to $D 2_{x}$, these values show greater deviation from each other within each sheared regime, especially for the low-shear transition regime. However, the overall trends are similar. Also, the corresponding values are lower for the particles of Type B, indicating a reduction in streamwise diffusion due to friction.

Profiles for $\langle\Delta y \Delta x\rangle$ are shown in figure 15 for experiment 3. The values are extremely small, and within the error limits of the measuring technique. This is a characteristic for all the cases considered.

Figure 16 shows the values of $D 1$, the trace of the diffusion tensor normalized by the root-mean-square fluctuation velocity. The measured values are reasonably consistent for all the regimes for the case of the particles of Type A. In the case of the particles of Type B, the results for the two larger exit width conditions are consistent for all three regimes, but values for the narrowest exit condition diverge for the low-shear and uniform-flow regimes. The values of the trace of the diffusion tensor normalized by the strain rate, $D 2$, are listed in table 2 .

Figures 16 and 14 are notably similar since the values of $D_{y y}$ are almost an order-ofmagnitude higher than $D_{x x}$ for all the regimes examined. This similarity is reflected in the values of $D 2_{y}$ and $D 2$ in table 2 . The anisotropy in the diffusion coefficient arises as a result of the anisotropy in the fluctuation velocities.

While a direct comparison of the measured diffusion coefficients with those predicted by the arguments based on kinetic theory (equation (4.3)) is inhibited by the inability to measure solid fractions in the flows, it is useful to make some comparisons using reasonable estimates for the solid fraction and the coefficient of restitution. The radial distribution function $g_{0}(v)$ is assumed to be equal to $\left(1-\left(v / v^{*}\right)\right)^{-2.5 v^{*}}$ (Lun \& Savage 1986 ), with the maximum shearable solid fraction $\nu^{*}$ equal to 0.64 . Using values of 0.9 
for the coefficient of restitution and 0.5 for the solid fraction, $D_{i i} / \sigma T^{1 / 2}$ (from (4.3)) is equal to 0.022 , and reduces to 0.0075 for a solid fraction of 0.56 . In comparison, in the moderate-shear regime in Experiment $3, D 1_{x}$ is equal to 0.035 . However, if the transverse diffusion coefficient $D_{x x}$ is normalized by the root-mean-square fluctuation velocity, a value of 0.019 is obtained. Both of these values are close to the value for $D 1_{i}$ (of the same order of magnitude) for an assumed solid fraction of 0.5 . However, if the trace of the diffusion tensor is normalized by the root-mean-square velocity (see figure 16), a value of 0.22 is obtained. Using the condition of isotropy, which is assumed in deriving (4.3), the kinetic theory value is 0.044 for a solid fraction of 0.5 and reduces sharply at higher solid fractions. The cause of the discrepancy may lie in the assumptions of isotropy in (4.3). The use of an isotropic radial distribution function to describe the wall-bounded sheared flows of the type encountered in the current experiments may not be appropriate. Furthermore, as previously noted, the current experiments do not belong to the rapid granular flow regime that is well represented by theory based on the kinetic theory of dense gases. Hence, while the measured diffusion coefficients conform to the trends predicted by the kinetic theory, it is apparent from the discussion above that the expression for diffusion coefficients derived from kinetic theory, (4.3), cannot be used to make quantitative predictions for the high-density flows examined here.

The marked anisotropy of the diffusive behaviour of the flows considered contrasts with the results of Scott \& Bridgewater (1976), as examined by Bridgewater (1980). In those experiments, the particles were $18.6 \mathrm{~mm}$ in diameter and the strain rates were lower than those considered in the present work. Furthermore, while both Savage \& Dai (1993) and Campbell (1993) report that their simulations indicate somewhat larger diffusion in the streamwise direction, the differences are not as large as those observed in this work. More importantly, even when the diffusion coefficients are normalized by the appropriate fluctuation velocities (figures 12,15), the difference between the nondimensionalized parameters is substantial. Campbell (1993) has suggested that the diffusional anisotropy is enhanced because the shear motion may introduce an internal structure that permits greater freedom of motion in preferred directions. Also, even though their streamwise fluctuation velocities are comparable in magnitude, the diffusion coefficients $D_{y y}$ are much lower in the uniform flow regime than in the lowshear transition regime. Obviously, the solid fraction plays a very important role here.

The coefficients of diffusion usually increase with the mean flow velocity in the uniform flow regime. This observation seems consistent with the fact that the fluctuation velocity components scale reasonably well with the local mean velocity. Hwang \& Hogg (1980) reported that their measurements for transverse diffusion in an inclined chute were comparable with a model in which the diffusion coefficient was composed of two parts: one proportional to the shear rate and the other a constant indicative of the 'intrinsic' random fluctuations of the particles. However, they did not indicate whether there was any relation between this intrinsic component and the mean flow velocity. Their measured transverse diffusion coefficients were of the same order as those measured in the current experiments in the moderate-shear regimes.

\section{Conclusion}

The current experiments used image processing methods to measure velocity profiles and diffusion coefficients for two-dimensional granular flows in a vertical channel with roughened walls. Both mean and fluctuation velocity profiles were measured. Three different types of particles were considered with three mean flow rates for each type. 
From the velocity profiles, and the diffusive behaviour, three flow regimes were identified for each flow: a central uniform-flow regime, a low-shear transition regime, and an outer moderate-shear regime. The measurements showed that the fluctuation velocities were anisotropic with the longitudinal components being about 2 to 2.5 times the transverse component. The transverse fluctuations showed a greater dependence on the shear rates than the streamwise components. Both fluctuation velocity components increased with the flow rate. For comparable shear rates, the fluctuation velocities were lower in the case of rough particles, indicating a damping of random fluctuation energy due to friction.

The diffusion coefficients increased with shear rate and fluctuation velocity. However, the proportionality factors depended on the shear regime. The diffusion coefficients were anisotropic, with the values in the streamwise direction being an order-of-magnitude higher. Finite fluctuation velocities and diffusive displacements were observed in the central uniform regime. Visual observations as well as the measured values indicated that the diffusive behaviour depended on the solid fraction. However, the solid fraction profiles were not measured. There was a reduction in diffusion coefficients for the case of rough particles.

A better understanding of the constitutive relations governing these flows would help explain the results obtained in this work. Also, future work should concentrate on methods to make local measurements of the solid fraction in such dense flows. Another subject of future work is the study of the influence of particle mixing on heat transfer rate, especially in regimes close to boundaries.

\section{REFERENCES}

AhN, H., Brennen, C. E. \& SABERSKy, R. H. 1991 Measurements of velocity, velocity fluctuations, density and stresses in chute flows of granular materials. Trans. ASME E: J. Appl. Mech. 58, $792-803$.

Brennen, C. \& Pearce, J. C. 1978 Granular material flow in two-dimensional hoppers. Trans. ASME E: J. Appl. Mech. 45, 43-50.

Bridgewater, J. 1980 Self diffusion coefficients in deforming powders. Powder Technol. 25, 129-131.

BUGGish, H. \& LofFelmanN, G. 1989 Theoretical and experimental investigation into local granular mixing mechanisms. Chem. Engng. Proc. 26, 193-200.

Campbell, C. S. 1989 The stress tensor for simple shear flow of a granular material. J. Fluid Mech. 203, 449-473.

Campbell, C. S. 1993 Self-diffusion in granular shear flows. J. Fluid Mech. (submitted).

Chapman, S. \& Cowling, T. G. 1971 The Mathematical Theory of Non-uniform Gases, 3rd edn. Cambridge University Press.

DAI, R. 1993 Granular flow studies through kinetic theory and numerical simulation approaches. $\mathrm{PhD}$ thesis, McGill University, Montreal.

DRAKE, T. G. 1991 Granular flow: physical experiments and their implications for microstructural theories. J. Fluid Mech. 225, 121-152.

EINSTEIN, A. 1956 In Albert Einstein, Investigations on the theory of the Brolinian movement (ed. R. Furth), chap. 1, §4, pp. 12-17. Dover.

Goodman, M. A. \& Cowin, S. C. 1971 Two problems in the gravity flow of granular materials. $J$. Fluid Mech. 45, 321-339.

HaFF, P. K. 1983 Grain flow as a fluid mechanical phenomenon. J. Fluid Mech. 134, 401-430.

Hsiau, S. S. \& HUNT, M. L. $1993 a$ Shear-induced particle diffusion and longitudinal velocity fluctuations in a granular-flow mixing layer. J. Fluid Mech. 251, 299-313.

Hsiau, S. S. \& HUNT, M. L. $1993 b$ Kinetic theory analysis of flow-induced particle diffusion and thermal conduction in granular material flows. Trans. ASME C: J. Heat Transfer 115, 541-548. 
Hui, K. \& HAFF, P. K. 1986 Kinetic grain flow in a vertical channel. Intl J. Multiphase Flow 12, 289-298.

Hunt, M. L., Hsiau, S. S. \& Hong, K. T. 1994 Particle mixing and volumetric expansion in a vibrated granular bed. Trans. ASME I: J. Fluids Engng 116, 785-791.

HWANG, C. L. \& HoGg, R. 1980 Diffusive mixing in flowing powders. Powder Technol. 26, 93-101.

Jenkins, J. T. \& Savage, S. B. 1983 A theory for the rapid flow of identical, smooth, nearly elastic, spherical particles. J. Fluid Mech. 130, 187-202.

Johnson, P. C. \& JACKSON, R. 1987 Frictional-collisional constitutive relations for granular materials, with application to plane shearing. J. Fluid Mech. 176, 67-93.

Liu, K. S., Kalos, M. H. \& Chester, G. V. 1974 Quantum hard spheres in a channel. Phys. Rev. A 10, 303-308.

Louge, M. Y. 1994 Computer simulations of rapid granular flows of spheres interacting with a flat, frictional boundary. Phys. Fluid. 6, 2253-2269.

Lun, C. K. K. \& SAvaGe, S. B. 1986 The effects of an impact velocity dependent coefficient of restitution on stresses developed by sheared granular materials. Acta Mech. 63, 15 44 .

Lun, C. K. K., Savage, S. B., Jeffrey, D. J. \& Chepurniy, N. 1984 Kinetic theories for granular flow: inelastic particles in Couette flow and slightly inelastic particles in a general flow field. J. Fluid Mech. 140, 223-256.

Nedderman, R. M. \& Laohakul, C. 1980 The thickness of the shear zone of flowing granular materials. Powder Technol. 25, 91-100.

NGUYEN, T. V., BrenNen, C. \& SABERSKy, R. H. 1979 Gravity flow of granular materials in conical hoppers. Trans. ASME E: J. Appl. Mech. 46, 529-535.

Nunziato, J. W., Passman, S. L. \& Thomas, J. P. 1980 Gravitational flows of granular materials with incompressible grains. J. Rheol. 24, 395-420.

Richman, M. W. \& Marciniec, R. P. 1991 Granular chute flows. In Recent Advances in Mechanics of Structured Continua, AMD-Vol. 117. ASME.

SAvAGE, S. B. 1979 Gravity flow of cohesionless granular materials in chutes and channels. J. Fluid Mech. 92, 53-96.

Savage, S. B. \& DaI, R. 1993 Studies of shear flows. Wall slip velocities, 'layering' and selfdiffusion. Mech. Mater. 16, 225-238.

SAvage, S. B. \& JefreeY, D. J. 1981 The stress tensor in a granular flow at high shear rates. J. Fluid Mech. 110, 255-272.

Scott, A. M. \& Bridgewater, J. 1976 Self-diffusion of spherical particles in a simple shear apparatus. Powder Technol. 14, 177-183.

Sherwood, T. K., Pigford, R. L. \& Wilke, C. R. 1975 Mass Transfer. McGraw Hill.

TAKAHASHI, H. \& YaNAI, H. 1973 Flow profiles and void fraction of granular solids in a moving bed. Powder Technol. 7, 205-214.

TAYLOR, E. D. \& HunT, M. L. 1993 Measurements of velocity fluctuations in a granular flow. In Powders \& Grains 93 (ed. C. Thornton). Balkema.

TAYLOR, G. I. 1953 Dispersion of soluble matter in solvent flowing slowly through a tube. Proc. $R$. Soc. Lond. A 219, 186-203.

TAYLOR, G. I. 1954 The dispersion of matter in turbulent flow through a pipe. Proc. R. Soc. Lond. A 223, 446-468.

Walton, O. R. \& Braun, R. L. 1986 Viscosity, granular temperature and stress calculations for shearing assemblies of inelastic, frictional disks. J. Rheol. 30, 949-980.

ZIK, O. \& Stavans, J. 1991 Self-diffusion in granular flows. Europhys. Lett. 16, 255-258. 